\title{
La eliminación de la lepra de las Américas: situación actual y perspectivas
}

\author{
Clovis Lombardi, ${ }^{1}$ Celina M. T. Martolli, ${ }^{2}$ \\ Simonne Almeida e Silva ${ }^{3}$ y Reinaldo E. Gil Suárez ${ }^{4}$
}

RESUMEN La lepra, enfermedad que antes evocaba una imagen sombría e inspiraba terror, ahora se puede curar gracias al esquema politerapéutico a base de rifampicina, clofazimina y dapsona que se ha venido usando desde 1981. En 1991 la Asamblea Mundial de la Salud, alentada por la eficacia de este régimen, fijó la meta de eliminar la enfermedad como problema de salud pública mundial y nacional para el año 2000. Esta meta, que equivale a reducir la prevalencia a menos de un caso por 10000 habitantes, no debe confundirse con la de erradicar la enfermedad, que implica interrumpir por completo su transmisión. La eliminación de la lepra es una meta asequible que dependerá del uso enérgico y a gran escala del régimen poliquimioterapéutico. El presente trabajo describe y examina las iniciativas que se han puesto en marcha en América Latina para lograr la meta y los resultados observados hasta el momento. También se exploran los factores que inciden en la factibilidad de erradicar la enfermedad.

En la actualidad, la lepra es una enfermedad curable (1-5) como resultado de los avances de la quimioterapia y, especialmente, de la utilización desde 1981 de la poliquimioterapia recomendada por la OMS $(6,7)$. En los últimos 15 años, la experiencia acumulada con este esquema terapéutico (que consiste en la administración de tres medicamentos —rifampicina, clofazimina y dapsona- para tratar los casos multibacilares o paucibacilares de acuerdo con la clasificación operacio-

\footnotetext{
Las solicitudes de separatas han de dirigirse a este autor a la siguiente dirección postal: Organización Panamericana de la Salud, SEN Lote 19, 70.800400, Brasilia DF, Brasil.

2 Universidad Federal de Goias, Instituto de Patología Tropical y Salud Pública, Departamento de Salud Colectiva, Goiania, Brasil.

3 Universidad Federal de Goias, Instituto de Patología Tropical y Salud Pública, Departamento de Salud Colectiva, Goiania, Brasil.

4 Organización Panamericana de la Salud, Brasilia, Brasil.
}

nal de la lepra recomendada por la OMS (8)) ha mostrado que este tratamiento es muy efectivo para curar la enfermedad; es aceptado por la mayoría de los pacientes; tiene efectos secundarios mínimos, y previene la aparición de cepas de Mycobacterium leprae resistentes a los medicamentos (9).

El estado actual de los conocimientos sobre la utilización de vacunas contra la lepra (10-14) no ha permitido emprender aún acciones de prevención primaria. Sin embargo, la consideración de la lepra como problema de salud pública y la introducción de la poliquimioterapia cambiaron diametralmente la imagen sombría que ofrecía la lucha contra la enfermedad en años recientes (9). El perfil epidemiológico de la lepra cambió notablemente en la mayoría de los países endémicos $(1,2)$ y su eliminación se convirtió en una meta factible $(7,15)$.
En 1991, la Asamblea Mundial de la Salud aprobó la resolución relativa a la eliminación de la lepra como problema de salud pública a nivel mundial y nacional en el año 2000 (1, 2). Esta decisión histórica de la comunidad internacional ha tenido una enorme repercusión, ha despertado el interés de los países en el control de la enfermedad y ha contribuido a aumentar el número de pacientes tratados con poliquimioterapia (16). Por otra parte, el término "eliminación", incluido en la definición de la meta, ha creado cierta confusión $(16,17)$. La eliminación no debe ser confundida con la erradicación, por cuanto esta última supone la interrupción completa de la transmisión, la reducción a cero de la incidencia y la desaparición total del agente causal (9) o, en otras palabras, la supresión de toda fuente de infección o infestación, de tal manera que, aun 
cuando no se tomen medidas preventivas de ninguna clase, la enfermedad no reaparezca $(18,19)$. La eliminación de la lepra como problema de salud pública es una meta menos ambiciosa, pues para considerar que esta se ha alcanzado la prevalencia de la enfermedad ha de reducirse a menos de 1 caso por 10000 habitantes $(1,20)$.

La principal estrategia para lograr la eliminación de la lepra ha sido la utilización de la poliquimioterapia de la OMS y la detección de casos $(4,21)$ mediante las siguientes medidas: la estratificación geográfica del problema sobre la base de los indicadores epidemiológicos y operacionales; el establecimiento de prioridades a partir de la estratificación; y el monitoreo y la evaluación sistemáticos de los indicadores epidemiológicos y operacionales (5). También se han fijado los requisitos esenciales para lograr esta meta, que pueden resumirse del siguiente modo: alta cobertura $(95 \%)$ con poliquimioterapia, gran número de tratamientos concluidos (90\%), diferencia entre el registro activo y la prevalencia estimada que no exceda $10 \%$ - producto de una buena detección de casos-, actualización y monitoreo permanente de un plan de eliminación, y movilización de recursos y apoyo político continuos (20). En síntesis, la eliminación de la lepra como problema de salud pública se logrará mediante el diagnóstico temprano y la administración oportuna a todos los enfermos de la poliquimioterapia recomendada por la OMS (1).

La utilización enérgica y masiva de la poliquimioterapia ofrece en la actualidad una oportunidad única para eliminar la lepra (1). Hasta la fecha, se han logrado avances notables para alcanzar esta meta. A principios de 1996 se habían registrado en todo el mundo 926259 casos, lo cual supone una reducción de la prevalencia de $82,7 \%$ respecto a 1985 y de $28 \%$ respecto a 1995 (22). El número de casos estimados también se ha reducido en $31 \%$ en relación con 1995 y en $77 \%$ cuando se compara con el notificado en $1991(22,23)$.

El presente trabajo tiene como objetivos describir y analizar los resultados obtenidos hasta el momento en Amé- rica Latina, en concreto, y en la Región, en general, con los esfuerzos realizados para alcanzar la meta de eliminar la lepra, su factibilidad y posibles escenarios, y las posibilidades de interrumpir la transmisión de la enfermedad.

\section{MATERIALES Y MÉTODOS}

Para realizar este trabajo se utilizaron los datos oficiales que anualmente aportan los sistemas de información de salud de los países de la Región a la OPS (24-26). Las tasas que se presentan se calcularon a partir de los datos de población oficiales de las Naciones Unidas (27). La selección de los países se basó en la información disponible y en el propósito de analizar diferentes escenarios epidemiológicos y respuestas operacionales para la aplicación del esquema de quimioterapia. Así, se han incluido algunos países que ya han alcanzado la meta de eliminación y otros que todavía no la han logrado y en los cuales vive la mayor parte de la población y de los enfermos de lepra de América Latina.

Los datos utilizados corresponden a tres momentos diferentes (1982, $1991 \mathrm{y}$ 1995) de la historia reciente de las acciones emprendidas contra la lepra, en especial en lo que atañe a la utilización de la poliquimioterapia. El año 1982 puede considerarse representativo de la etapa previa a la utilización de la poliquimioterapia; en 1991 esta se comenzó a administrar a gran escala y 1995 traduce de forma aproximada la situación actual. Para América Latina en su conjunto, se compararon los dos últimos momentos del proceso, porque no se disponía de datos precisos de 1982.

Entre los países de la Región existen algunas diferencias en cuanto a la información disponible sobre la definición de caso de lepra y el tiempo que los casos permanecen en los registros aun después de no estar ya en control por haber abandonado el tratamiento antes de cumplirse 12 meses -la OMS pide que estos casos se eliminen de los registros-, lo cual puede influir en las estimaciones de la prevalencia de la enfermedad. También pueden existir diferencias en la calidad de los sistemas de obtención de datos. No obstante, las definiciones y la calidad se mantuvieron estables en el período estudiado. Por ello, es posible comparar datos de distintos períodos tanto de un mismo país como de la Región en su conjunto.

El análisis de datos consistió en el cálculo de las diferencias porcentuales entre las cifras de prevalencia de los diferentes momentos analizados. Suponiendo que la variación de la prevalencia se mantuvo constante en cada período, para calcular la media anual de variación de la prevalencia se calculó la media geométrica de la prevalencia anual de acuerdo con la siguiente fórmula:

$\begin{aligned} \text { Media de la variación anual } & =\frac{n}{-\sqrt{1-p}}\end{aligned}$

siendo $n$ el número total de años en el período considerado y $p$, la variación porcentual de la prevalencia en todo el período (28). En esta fórmula se consideraron los 9 años del intervalo entre 1982 y 1991 y los 4 años transcurridos entre 1991 y 1995.

\section{RESULTADOS}

Cuando se comparan las tasas de prevalencia de 1982 y 1991 en los países seleccionados (figura 1, cuadro 1), se observa una disminución en la mayoría de ellos, con proporciones que oscilan entre 14 y $84 \%$. En este período, el ritmo promedio de reducción de la prevalencia puede considerarse bajo en la mayoría de los países, si bien el Brasil es el único país en que aumentó la prevalencia durante este período.

A partir de 1991, la prevalencia siguió disminuyendo, pero a un ritmo mucho mayor en casi todos los países. En la República Dominicana el ritmo se redujo, puesto que ya se había logrado una reducción importante de la prevalencia entre 1982 y 1991. En el Brasil, la tendencia de la prevalencia se modificó y se redujo más de $50 \%$ en 4 años, lo cual equivale a un ritmo anual de reducción de $16 \%$. 
FIGURA 1. Prevalencia de lepra en varios países de América Latina en 1982, 1991 y 1995

Tasa de prevalencia por 10000 habitantes

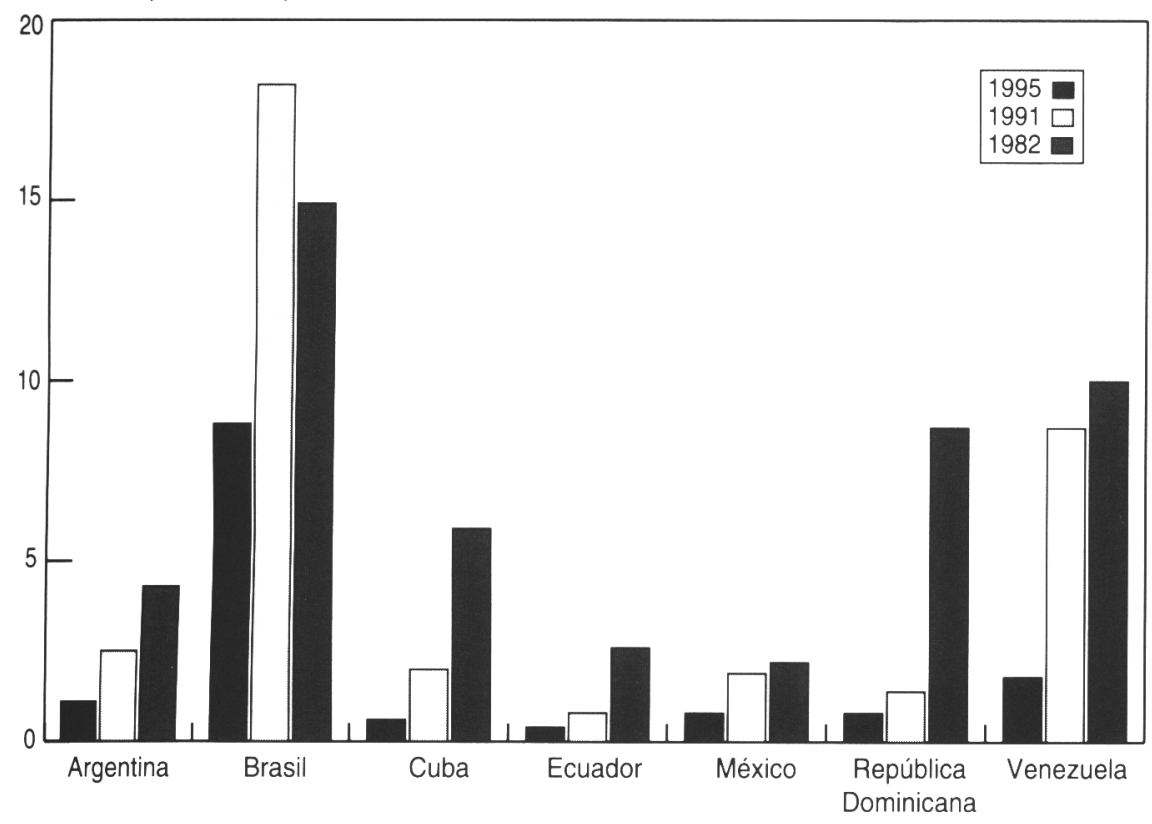

Entre 1991 y 1995, la prevalencia en América Latina (figura 2) se redujo de 8,1 a 3,6 por 10000 habitantes, es decir, disminuyó $55,6 \%$, lo que equivale a un descenso anual medio de $18,4 \%$. Solamente en el Brasil la prevalencia era mayor de 3 por 10000 habitantes a finales del período, momento en el cual muchos países ya habían logrado eliminar la enfermedad de su territorio nacional.

El marcado ascenso del ritmo de reducción de la prevalencia observado entre 1991 y 1995 se relaciona con el aumento de la cobertura de la poliquimioterapia, que ascendió de 23,9\% en 1991 a 75,6\% en 1995 (figura 3). Este cambio supone un aumento de la cobertura de $51,7 \%$ y puede compararse con las coberturas de la mayor parte de los países de la Región, que sobrepasaban 75\%. En los países seleccionados (cuadro 2) destaca el aumento de la cobertura en el Brasil, México y Venezuela. A pesar de que la cobertura era elevada en los países res-

CUADRO 1. Porcentaje de variación de la prevalencia de la lepra en países seleccionados de América Latina en el período de 1982 a 1995

\begin{tabular}{|c|c|c|c|c|}
\hline \multirow[b]{3}{*}{ País } & \multicolumn{4}{|c|}{ Variación de la prevalencia (\%) } \\
\hline & \multicolumn{2}{|c|}{$1982-1991$} & \multicolumn{2}{|c|}{$1991-1995$} \\
\hline & Período $^{1}$ & Anual $^{2}$ & Período $^{1}$ & Anual $^{2}$ \\
\hline Argentina & $-41,9$ & $-5,9$ & $-56,9$ & $-18,6$ \\
\hline Brasil & $+22,2$ & $+2,3$ & $-51,6$ & $-16,6$ \\
\hline Cuba & $-66,1$ & $-11,3$ & $-70,0$ & $-26,0$ \\
\hline Ecuador & $-69,2$ & $-12,3$ & $-50,0$ & $-15,9$ \\
\hline México & $-13,6$ & $-1,6$ & $-57,9$ & $-19,4$ \\
\hline República Dominicana & $-83,9$ & $-18,4$ & $-42,9$ & $-13,1$ \\
\hline Venezuela & $-33,0$ & $-4,4$ & $-73,1$ & $-28,0$ \\
\hline
\end{tabular}

${ }^{1}$ Diferencia porcentual entre los años extremos del período.

${ }^{2}$ Media geométrica de la variación anual en el período.

tantes, en el Ecuador y Cuba se mantuvo más o menos constante, en la Argentina aumentó, y en la República Dominicana descendió, aunque nunca a menos de $90 \%$.

Estos indicadores muestran que en los últimos años se ha producido en la Región una modificación importante de la situación de la enfermedad. Cuando se comparan las tasas de detección de casos nuevos de lepra en América Latina de 1992 y 1995, se observa que se mantienen prácticamente inalteradas y que las prevalencias son casi idénticas en los dos períodos comparados.

\section{DISCUSIÓN}

En la Región de las Américas la lepra no es endémica en Estados Unidos, Canadá ni Chile y recientemente se ha eliminado como problema de salud pública en la mayor parte de los países del Caribe de habla inglesa. De esta forma, el área endémica de la lepra en la Región se circunscribe prácticamente a los países de América Latina.

En comparación con otras regiones de la OMS, hasta fines de la década de los ochenta los logros habían sido menores en la Región de las Américas (2, 29) en cuanto a la velocidad del proceso de eliminar la lepra desde la introducción de la poliquimioterapia. Ello se atribuye a la existencia inicial de requisitos para la aplicación de la poliquimioterapia demasiado estrictos e inapropiados; la inadecuada capacitación de la gerencia de los programas en el marco de los sistemas de salud; la aplicación en algunos países de normas técnicas tendientes a contar en las cifras de prevalencia a enfermos ya curados, como consecuencia de la creencia tradicional de que la lepra no se cura; así como la renuencia de algunos profesionales de la salud a aceptar los esquemas de la poliquimioterapia $(30,31)$.

La reducción de la prevalencia de la lepra observada en América Latina en el período comprendido entre 1982 y 1991, aunque se haya producido con cierta lentitud, debe ser el resultado de las acciones de los programas de control que se venían ejecutando 
FIGURA 2. Prevalencia de lepra en los países de América Latina en 1991 y 1995

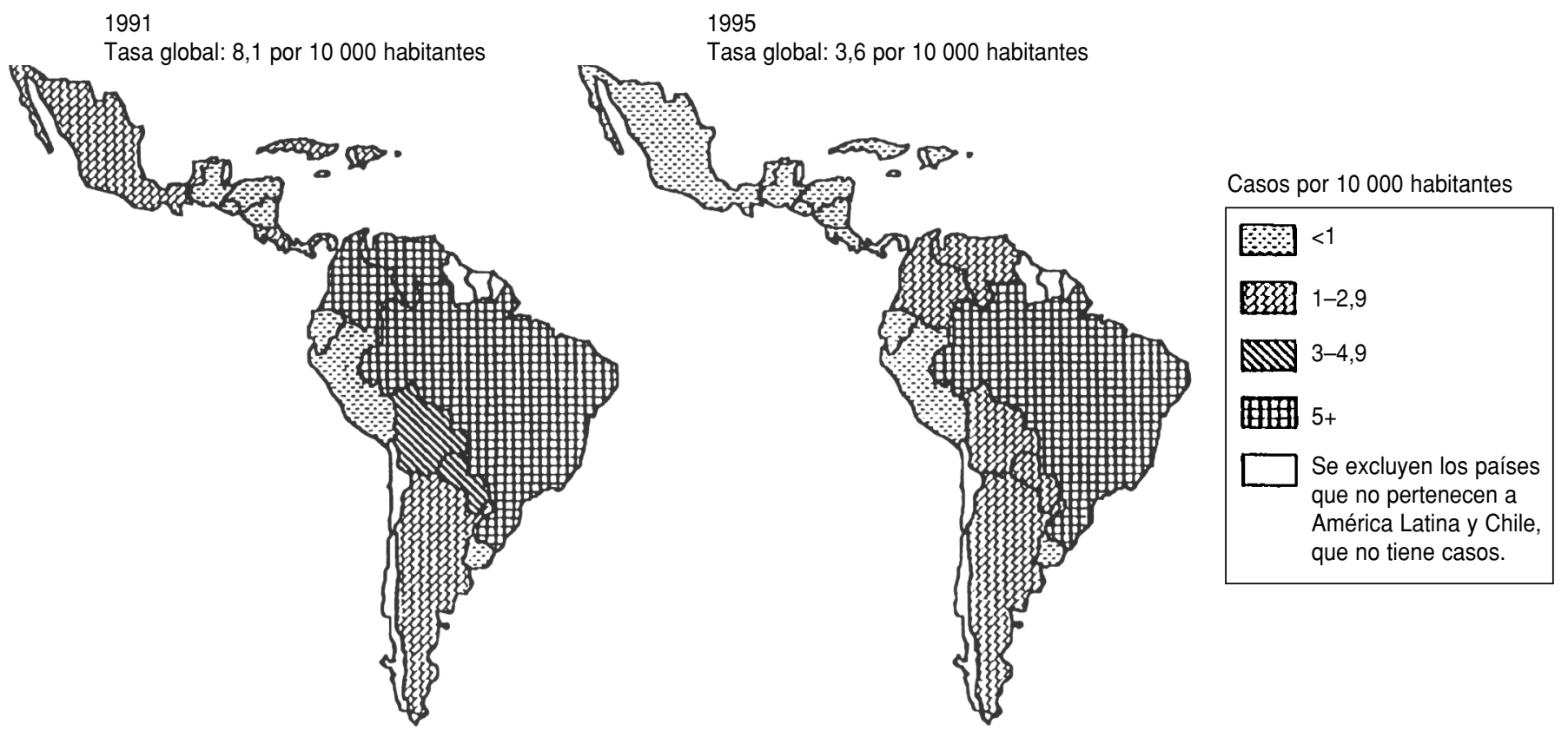

desde hacía varios años y de la limitada implantación de la poliquimioterapia, que comenzó a utilizarse en ese período en diferentes momentos y con intensidades distintas según los países. El ritmo de reducción observado muestra que los esquemas de tratamiento utilizados antes de la poliquimioterapia - que no garantizaban la curación de la enfermedad en un período definido - no lograban avances importantes en la reducción de la prevalencia y, por lo tanto, constituían un importante escollo para la meta de eliminación.

El aumento de la prevalencia observado en el Brasil en el período de 1982 a 1991 puede considerarse producto de factores operacionales, tales como el aumento de la búsqueda de casos y la descentralización de las acciones, y no de un ascenso verdadero del nivel de endemicidad de la lepra, y coincidió con el fortalecimiento del programa para el control de la enfermedad y el inicio de la utilización de la poliquimioterapia en 1986. En 1991 se celebró en México la Conferencia para el Control de la Lepra, en la cual los países adoptaron recomendaciones técnicas, se obtuvo el compromiso político y se definió el mandato para que la OPS elaborara el Plan Regional de Eliminación (24). Dicho Plan, que comenzó a ejecutarse en 1992, tiene como estrategia central la expansión masiva de la poliquimioterapia de la OMS, junto con la detección temprana de casos. Con tales propósitos, se definieron y comenzaron a poner en práctica las siguientes estrategias de apoyo: reforzamiento de la capacidad gerencial en los niveles nacional y subnacional, estratificación epidemiológica y operacional, y elaboración y ejecución de planes de eliminación en los niveles nacional y subnacional. Para poner en marcha estas estrategias, se programaron y llevaron a cabo actividades de capacitación del personal a nivel regional, nacional y subnacional. Por añadidura, se garantizó el suministro gratuito de los medicamentos específicos de la poliquimioterapia (31) en forma de paquetes con calendario (blister packs), medida que facilitó considerablemente su utilización como esquema terapéutico estandarizado en las condiciones de las unidades de atención primaria de salud.
A partir de 1992, la ejecución del Programa Regional de Eliminación de la Lepra lanzado por la OPS/OMS, junto con la utilización a gran escala de la poliquimioterapia y las actividades de apoyo que se introdujeron, ha desembocado en el aumento del ritmo de reducción de la prevalencia de la lepra en muchos países. Con diferencias regionales importantes $(32,33)$, en el Brasil se modificó la tendencia y la prevalencia de la enfermedad se redujo más de $50 \%$ en el período, lo que representó un descenso anual promedio de $17 \%$. Debe destacarse que en este país se concentra un elevado porcentaje de los casos de lepra de la Región y, por ello, los resultados van a tener una influencia muy importante en los de América Latina en su conjunto $(31,34)$.

En relación con la estabilidad observada en la detección de casos nuevos en el período de 1991 a 1995, puede considerarse que la influencia de la poliquimioterapia no se hace patente de forma inmediata en las tendencias de detección de casos de la enfermedad. El período de incubación de la lepra suele ser largo (5 años en promedio), y ello 
FIGURA 3. Cobertura de pacientes de lepra con poliquimioterapia en los países de América Latina en 1991 y 1995

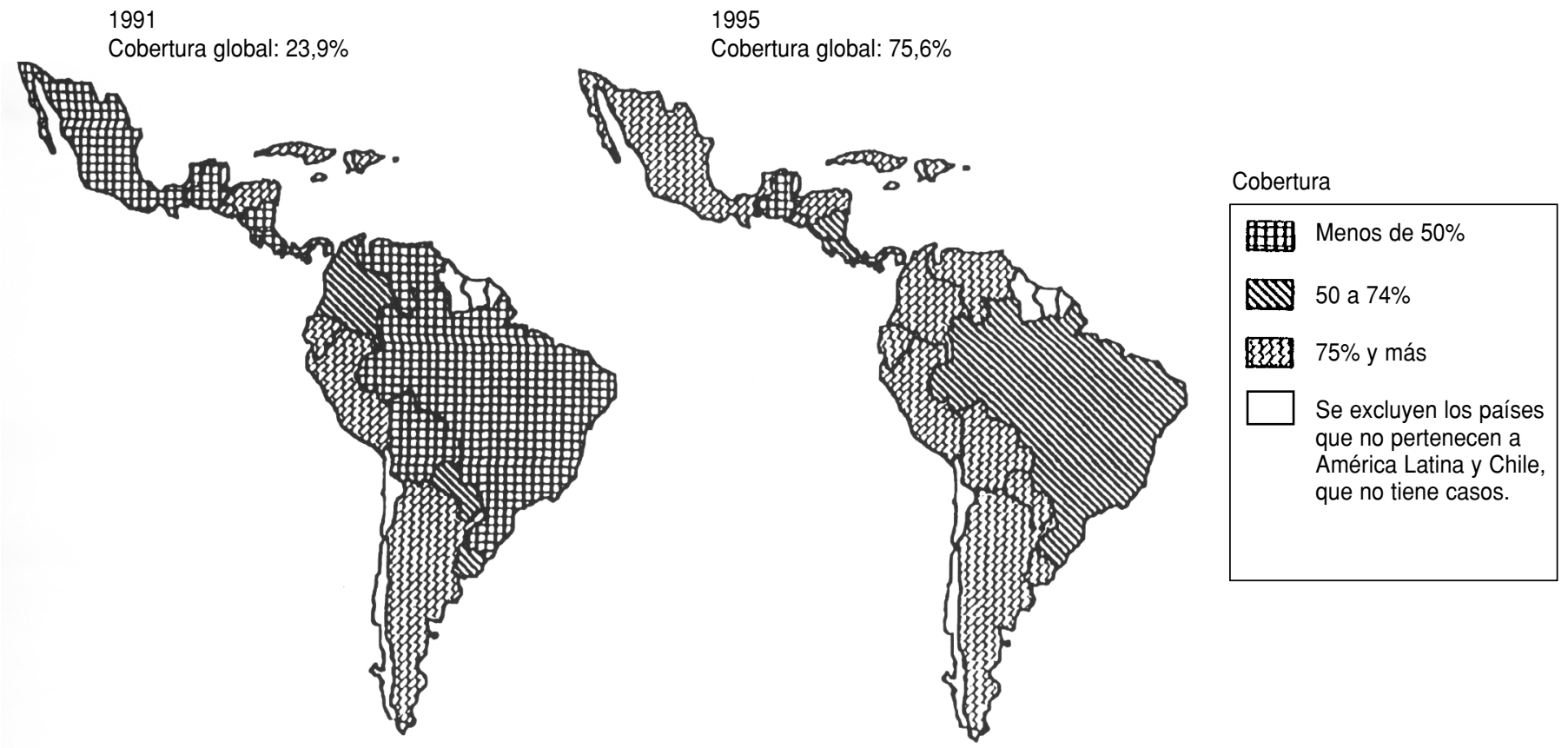

explica que se continúe diagnosticando casos por períodos más o menos largos en personas infectadas en años anteriores. Por otra parte, se espera que a mediano plazo la eliminación paulatina de fuentes de infección por medio de la poliquimioterapia influya en la transmisión y, por ende, en la detección de casos nuevos $(9,35,36)$.

De acuerdo con los resultados expuestos, se puede afirmar que la prevalencia de lepra tiende a disminuir en la Región de las Américas y, por consiguiente, la meta de eliminar la enfer- medad puede considerarse factible si se tiene en cuenta que, en virtud de las proyecciones de la población regional (27), la prevalencia de 1 caso por 10000 habitantes en América Latina se alcanzaría con 52000 enfermos. Ello sería posible si se mantuviera un ritmo de reducción similar al que se ha venido observando en los últimos 4 años. Con este número de casos, la tasa de prevalencia para toda la Región será de 0,6 casos por 10000 habitantes.

En un futuro inmediato, las acciones de eliminación se desarrollarán en es-

CUADRO 2. Cobertura con poliquimioterapia de personas con lepra en países seleccionados de América Latina, 1991-1995

\begin{tabular}{lrcc}
\hline \multirow{1}{*}{ País } & \multicolumn{3}{c}{ Cobertura (\%) } \\
\cline { 2 - 4 } & 1991 & 1995 & Diferencia \\
\hline Argentina & 87,0 & 96,1 & $+9,1$ \\
Brasil & 18,0 & 73,6 & $+55,6$ \\
Cuba & 95,0 & 94,7 & $-0,3$ \\
Ecuador & 100,0 & 100,0 & $+41,4$ \\
México & 36,0 & 77,4 & $-7,0$ \\
República Dominicana & 99,0 & 92,0 & $+37,0$ \\
Venezuela & 16,0 & 53,0 & \\
\hline
\end{tabular}

cenarios más o menos favorables, dependiendo de la evolución de factores contextuales de variada naturaleza: demográficos y socioeconómicos; de los sistemas de salud, y de los programas de control de enfermedades endémicas (31). En este sentido, no deben esperarse modificaciones importantes en la situación socioeconómica de América Latina, aunque ha de continuar el aumento observado en la proporción de la población urbana, el cual representa un factor importante que debe tenerse en cuenta en la planificación de las acciones. Los sistemas de salud deben proseguir el proceso de descentralización, y los componentes del proceso de controlar y eliminar la lepra han de ajustarse en este proceso a una estrategia general integrada por tres elementos: descentralización, municipalización e integración. En dicho proceso, las acciones que se emprendan no han de ejecutarse exclusivamente en el nivel de la atención primaria de salud, sino que deben ser responsabilidad de las autoridades locales de ese nivel (es decir, del municipio y del distrito) y ejecutarse en el marco de las actividades básicas de salud. 
Por otro lado, el mantenimiento de las acciones futuras de control de la lepra va a depender en gran medida de lo que se logre avanzar en la consolidación de esa estrategia. La reducción acelerada de la endemicidad irá aumentando la importancia de la estratificación a niveles cada vez más periféricos del sistema de salud, en los cuales han de aprovecharse todas las posibilidades que ofrecen las técnicas avanzadas de geoprocesamiento computadorizado, con objeto de identificar territorios o poblaciones en alto riesgo. Ello permitirá concentrar los esfuerzos y lograr el impacto deseado con una adecuada utilización de los recursos. En este contexto, la identificación de áreas geográficas o de poblaciones en situaciones especiales (por ejemplo, la probable existencia de casos sin diagnosticar o de dificultades excepcionales para aplicar la poliquimioterapia recomendada por la OMS) debido a problemas de acceso geográfico o social, o por carencia de una mínima infraestructura de salud, hacen que se necesiten intervenciones especiales que permitan acelerar el proceso hacia la eliminación.

Según los pronósticos emitidos, será necesario colocar cada vez más en un primer plano las perspectivas del trabajo futuro en una situación de baja prevalencia (37). En otras palabras, en la etapa posterior a la eliminación habrá que tener en cuenta que alcanzar una prevalencia menor de 1 caso por 10000 habitantes puede no significar el fin de las acciones contra la lepra, lo cual obligaría a diseñar y emprender nuevas estrategias $(15,20)$. Es preciso subrayar la necesidad de obrar anticipadamente y de programar la solución de los problemas propios de la nueva etapa (35).
En esta nueva etapa será necesario conocer con certeza la situación de la enfermedad, y en ello son de capital importancia las actividades de vigilancia epidemiológica intensiva; las acciones para descartar la existencia de casos no diagnosticados y la posibilidad de realizar estudios específicos en áreas silenciosas (en las cuales existieron focos importantes de la enfermedad).

Otro aspecto de vital importancia en la actualidad es la consolidación del proceso que ha llevado a la eliminación de la lepra como problema sanitario, la cual está directamente relacionada con la capacidad de dar respuesta al siguiente interrogante: ¿qué clase de servicios pueden mantenerse y a qué costo, teniendo en cuenta que se trata de una enfermedad de baja endemicidad y que está desapareciendo lentamente? (16). A este respecto puede señalarse que muchos programas integrados están demostrando que las acciones acometidas pueden ser efectivas cuando se ejecutan en los servicios generales de salud, aunque en los niveles central e intermedio es preciso mantener las funciones de capacitación, supervisión y referencia (16). Por otra parte, debe destacarse en esta etapa la importancia que reviste la investigación epidemiológica de todo caso nuevo detectado $(37,38)$, con objeto de identificar la posible fuente de infección, así como otros casos que puedan haber surgido a partir de la misma fuente o que sean secundarios al caso índice. La capacitación sistemática del personal de salud, el establecimiento de centros centinela, la referencia especializada, la garantía del suministro de medicamentos y el estudio detallado de todo caso nuevo detectado pueden considerarse aspectos básicos del tra- bajo que ha de realizarse en esta etapa, que deberá desarrollarse sobre la base de la estratificación epidemiológica reciente, la cual, a su vez, habrá de actualizarse periódicamente.

El objetivo final del control de la lepra es interrumpir su transmisión. Todo parece indicar que se continuará avanzando hacia este objetivo (36). En la actualidad, el elemento esencial para influir sobre la transmisión es el diagnóstico temprano, seguido de inmediato por el tratamiento eficaz de los enfermos (5, 7-9, 37, 39, 40). Para lograrlo, deben tenerse en cuenta mecanismos tales como la estratificación basada en el criterio de riesgo en las comunidades, la identificación y el seguimiento de personas en alto riesgo individual de enfermar, y la búsqueda activa de casos. Para alcanzar el objetivo de interrumpir la transmisión es de esperar que pueda contarse con tecnologías aún no disponibles, entre las que pueden incluirse vacunas eficaces, esquemas cortos de tratamiento y técnicas de laboratorio que permitan identificar la infección o la enfermedad en estadio preclínico.

A la luz de la situación actual y teniendo en cuenta las proyecciones inmediatas, puede considerarse factible eliminar la lepra como problema de salud pública en la Región de las Américas. Con tal fin es necesario comenzar a planificar las actividades que deberán emprenderse en la etapa posterior a su eliminación, especialmente en lo que se refiere a confirmar la eliminación alcanzada y a mantener el proceso. Los resultados alcanzados y los esperados para el año 2000 permiten idear nuevas metas que tengan por objetivo final interrumpir la transmisión de la enfermedad y erradicarla.

\section{REFERENCIAS}

1. Organización Mundial de la Salud. Guía para la eliminación de la lepra como problema de salud pública. Ginebra: OMS; 1995. (Documento WHO/LEP/95.1.).

2. Noordeen SK. Eliminación de la lepra como problema de salud pública. Una perspectiva global. Informe de la Conferencia OPS/OMS sobre la Eliminación de la Lepra de las Américas, Brasilia, 27 a 29 de mayo de 1996. (Documento mimeografiado).

3. Summers A. Leprosy for field staff. London: Talmilep; 1993.
4. World Health Organization. Global strategy for the elimination of leprosy as a public health problem. Geneva: WHO; 1996. (Documento WHO/ LEP/96.7).

5. Gil R. Notas sobre la epidemiología de la lepra. Washington, DC: Organización Panamericana 
de la Salud; 1989. (Documento OPS/OMS, PNSP 89-42).

6. Organización Mundial de la Salud. Quimioterapia de la lepra para los programas de lucha: informe de un grupo de estudio de la OMS. Ginebra: OMS; 1982. (Serie de Informes Técnicos No. 675).

7. Organización Mundial de la Salud. Quimioterapia de la lepra: informe de un grupo de estudio de la OMS. Ginebra: OMS; 1994. (Serie de Informes Técnicos No. 847).

8. Organización Mundial de la Salud. Comité de Expertos de la OMS en Lepra: sexto informe. Ginebra: OMS; 1988. (Serie de Informes Técnicos No. 768).

9. Noordeen SK. Eliminating leprosy as a public health problem. Is the optimism justified? World Health Forum 1996;17:109-108.

10. Convit J, Sampson C, Zúñiga M, Smith $P G$ Plata J, Silva J, et al. Immunoprophylactic trial with combined Mycobacterium leprae/BCG vaccine against leprosy: Preliminary results. Lancet 1992;339:446-450.

11. Fine PEM. BCG vaccination against tuberculosis and leprosy. Br Med Bull 1988;44:691-703.

12. Convit J, Smith PG, Zúñiga M, Sampson $C$, Ulrich M, Plata J, et al. A BCG vaccination protects against leprosy in Venezuela: A casecontrol trial. Int J Lepr 1993;61:185-191.

13. Lombardi C, Pedrazzani ES, Pedrazzani JC, Filho PF, Zicker F. Protective efficacy of BCG against leprosy in São Paulo. Bull Pan Am Health Organ 1996;30:24-30.

14. Rodríguez MLO, Silva SA, Neto JCA, Andrade ALLS, Martelly CMT, Zicker F. Protective effect of intradermal BCG against leprosy: A case control study in central Brasil. Int J Lep 1992;60:335-339.

15. World Health Organization. Report of the International Conference on Leprosy. Hanoi, Vietnam, July 4-7, 1994. Geneva: WHO; 1994. (Documento WHO/CTD/LEP/94.5)

16. Feenestra $P$. The optimism is both an asset and a liability. World Health Forum 1996; 17:125-126.
17. Fine PEM. A more balanced view is needed. World Health Forum 1996;17:120-122.

18. Last JM. A dictionary of epidemiology. 2a ed. New York: Oxford University Press; 1988.

19. Soper FL. El concepto de erradicación de las enfermedades transmisibles. Bol Oficina Sanit Panam 1957;42:1-5

20. World Health Organization. Elimination of leprosy: Questions and answers. Geneva: WHO; 1996. (Documento WHO/LEP/96.4).

21. Roxas MG. Optimism in the Philippines. World Health Forum 1996;17:119-120.

22. World Health Organization. Progress towards the elimination of leprosy as a public health problem. Weekly Epidemiol Rec 1996; 71:149-156

23. World Health Organization. Progress towards the elimination of leprosy as a public health problem. Weekly Epidemiol Rec 1995; 70:177-184.

24. Lombardi C. Situación epidemiológica de la lepra en 12 países de América Latina y el Caribe, 1980-1983. Bol Oficina Sanit Panam 1988;105:20-33.

25. Organización Panamericana de la Salud. Informe de la Conferencia para el control de la lepra en las Américas. México, DF: OPS; 1991. (Documento PNSP92-13).

26. Organización Panamericana de la Salud. Situación de la lepra en los países de América Latina, 1995. Lepra al día 1996;1:3.

27. Fondo de Población de las Naciones Unidas. Estado de la población mundial, 1992. Nueva York: Naciones Unidas; 1992.

28. Santos JLF, Levy MSF, Szmerecsanyai T. Dinamica da população: teoria, métodos e técnicas de análise. São Paulo: Queiroz TA; 1991. p. 130.

29. Motta CP, Zúñiga MG. Time trends of Hansen's disease in Brazil. Int J Lep 1990; 58:453-461.

30. Becx-Bleumink M. It can be done but not so quickly. World Health Forum 1996;17:132-135.

31. Organización Panamericana de la Salud. La eliminación de la lepra de las Américas: situación actual y perspectivas. Informe de la Conferencia OPS/OMS sobre la eliminación de la lepra de las Américas. Brasilia: OPS; 1996. (Documento mimeografiado).

32. Martelli CMT, Andrade ALSS, Grossi MAF, Leboeuf MAA, Lombardi C, Zicker F. Changes in leprosy clinical pattern after multidrug therapy implementation. Int J Lep 1995; 63:95-97.

33. Penna GO. Hanseníase: epidemiologia e controle. Rev Soc Bras Med Trop 1994;27(Supl III):37-44.

34. Vázquez FA, Varela NN, Antola MC, Wand del Rey MML, Leguizamon OR. Hansen's disease in Mercosur. Acta Leprol 1996;10:79-84.

35. Smith WCS. Optimism justified but realism indispensable. World Health Forum 1996;17: 122-124.

36. Lechat MF. The effort itself is already more than justified. World Health Forum 1996;17: 139-142.

37. Gil RE, Ramírez R, Santín M, Lombardi C. Situación actual de la lepra en Cuba: ¿será factible la interrupción de la transmisión? Hansen Int 1996;21:34-45.

38. Andrade VLG, Sabrosa PC, Araujo AJG. Fatores associados ao domicílio e a família na determinação da hanseníase, Rio de Janeiro, Brasil. Cad Saude Publica 1994;10(supl 2): 281-292.

39. Gil R, Hernández O, De Rojas V. Programa de control de lepra para el médico de la familia. 2a ed. La Habana: Ministerio de Salud Pública; 1994.

40. Valdés LE, Orozco MI, Gil R. Guía para el control de foco de un caso de lepra. La Habana: Ministerio de Salud Pública; 1995.

Artículo recibido el 11 de diciembre de 1996 y aceptado para publicación, tras revisión, el 1 de mayo de 1998.
ABSTRACT Eliminating leprosy from the
Americas: Current situation
and future perspectives
Leprosy, a disease that used to be shrouded in darkness and fear, can now be cured thanks to a multidrug treatment schedule with rifampicin, clofazimine, and dapsone which has been in use since 1981. In 1991 the World Health Assembly, enouraged by the efficacy of this treatment regimen, established the goal of eliminating the disease as a public health problem globally and nationally by the year 2000 . This goal, which calls for reducing disease prevalence to less than one case per 10000 inhabitants, should not be confused with the goal of eradicating the disease, which implies a complete interruption of its transmission. Eliminating leprosy is an attainable goal which will depend on the forceful and massive use of the multidrug treatment regimen. This paper describes and discusses the various initiatives that have been launched in Latin America for the purpose of achieving this goal and the results obtained so far. It also explores the factors that impact on the feasibility of eradicating the disease. 